\title{
Thromboelastography With Platelet Mapping is Not an Effective Measure of Platelet Inhibition in Patients With Spontaneous Intracerebral Hemorrhage on Antiplatelet Therapy
}

Helena Lam ${ }^{1}$, Nakul Katyal ${ }^{2}$, Catherine Parker ${ }^{3}$, Prashant Natteru ${ }^{4}$, Premkumar Nattanamai ${ }^{2}$, Christopher R. Newey ${ }^{5}$, Chadd K. Kraus ${ }^{6}$

1. University of Missouri School of Medicine, Columbia, USA 2. Department of Neurology, University of Missouri, Columbia, USA 3. Emergency Medicine, University of Missouri, Columbia, USA 4. Department of Neurology, University of Mississippi Medical Center, Jackson, USA 5. Neurology, Cleveland Clinic Ohio, Akron, USA 6. Emergency Medicine, Geisinger Health System, Danville, USA

$\square$ Corresponding author: Nakul Katyal, katyal.nakul@gmail.com

Disclosures can be found in Additional Information at the end of the article

\section{Abstract}

Thromboelastography with platelet mapping (TEG-PM) is a modality to measure platelet function, especially in patients taking antiplatelet medications. It consists of two components: arachidonic acid (AA), which is sensitive to aspirin, and adenosine diphosphate (ADP), which is sensitive to clopidogrel. In patients with spontaneous intracerebral hemorrhages (sICH), the clinical interpretation of platelet mapping is unclear. The objective of this study was to evaluate TEG-PM in patients with sICH on aspirin and/or clopidogrel who receive platelet transfusions. This study was an IRB-approved, retrospective case-control study over three years at an academic medical center. Adult patients with sICH were included if they had an admission computed tomography head (CTH) and platelet mapping followed by a repeat platelet mapping and CTH post platelet transfusion. A threshold of 50\% inhibition was used as the benchmark for both ADP and AA inhibition. Around 248 subjects with sICH were identified, and 107 were excluded for incomplete documentation, leaving 141 for analysis. Of these, nine met our inclusion criteria. No statistical significance was found on the antithrombotic effects of aspirin or clopidogrel on TEG-PM ( $\mathrm{p}=1.00$ for both). Sensitivity and specificity of TEG-PM for clopidogrel was $100 \%$ and $42.9 \%$, respectively, and $80 \%$ and $0 \%$, respectively, for aspirin. Platelet transfusion did not significantly change AA or ADP inhibition ( $p=1.00)$. Hemorrhagic expansion on CTH was not associated with a decrease AA or ADP inhibition ( $p=1.00)$. TEG-PM is not an effective measure of platelet inhibition in sICH patients who were on antiplatelet medications and is not a reliable measurement following platelet transfusion.

Received 04/05/2018

Review began 04/10/2018

Review ended 04/16/2018

Published 04/22/2018

C) Copyright 2018

Lam et al. This is an open access article distributed under the terms of the Creative Commons Attribution License CC-BY 3.0., which permits unrestricted use, distribution, and reproduction in any medium, provided the original author and source are credited.
Categories: Internal Medicine, Neurology, Other

Keywords: intracerebral hemorrhage, platelet transfusion, platelet dysfunction, thromboelastography with platelet mapping

\section{Introduction}

Spontaneous intracerebral hemorrhage (sICH) is a devastating form of stroke that accounts for $6.5 \%$ to $19.6 \%$ of all strokes [1]. It has a $44 \% 30$-day mortality rate with half of the deaths occurring in the first 48 hours. Greater hematoma volume and expansion are significant 
indicators of poorer prognosis [2]. sICH in the setting of anticoagulation, such as with warfarin, has predictable monitoring with an international normalized ratio (INR) [3]. However, treating sICH in the setting of antiplatelet therapy (APT) is less predictable. A systematic review in 2010 showed that patients who were taking APT had a 27\% increased odds of death from sICH after one month compared to those not taking antithrombotic drugs [4]. Similarly, in a Finnish study, regular aspirin (ASA) use (median $250 \mathrm{mg}$ ) was associated with significant hematoma enlargement during the first week after sICH and two-fold increased mortality within the first three months [5]. A method to measure platelet function in sICH is desirable. Platelet transfusions are commonly used for APT reversal in the setting of active bleeding in sICH particularly if platelets $<100,000$ or if there is a need for emergent neurosurgical intervention [6]. However, platelet transfusion has not been found to reduce mortality [7]. A multi-center randomized trial (i.e., platelet transfusion versus standard care after acute stroke due to spontaneous cerebral hemorrhage associated with antiplatelet therapy (PATCH)) evaluated platelet transfusion within six hours from sICH for those on APT and found that there was an increased odds of death at three months $(\mathrm{OR}=2.05,95 \% \mathrm{CI}: 1.18-2.56 ; \mathrm{p}=0.01)$ compared to the group who had received no platelet transfusion. The results of this study are significant and suggest that caution should be used prior to platelet transfusion for antithrombotic reversal [8]. There are multiple different platelet functions tests currently available, each with limitations [9].

Thromboelastograph ${ }^{\circledR}$ Platelet Mapping ${ }^{\mathrm{TM}}$ (TEG-PM) (Haemoscope Corporation, Niles, IL, USA) is a newer modality to measure platelet function. It is a global hemostasis test that is additionally able to monitor antiplatelet inhibition of arachidonic acid (AA) and adenosine diphosphate (ADP) [10]. Unfortunately, these markers are abnormal in brain injured patients regardless of antiplatelet use [11]. Further studies are needed to determine its reliability particularly in patients with sICH. Given the results of the PATCH trial along with our local use of TEG-PM, we evaluated the following: 1) the sensitivity and specificity of the platelet assay portion of TEG-PM in relation to antithrombotic usage in the brain-injured patient, and 2) the efficacy of the platelet assay portion of TEG-PM in response to platelet transfusion and ICH outcome.

\section{Materials And Methods}

In this retrospective case-control study, data from December 1, 2012 to October 1, 2015 was retrieved from an electronic medical records (Cerner, North Kansas City, MO) database from an academic, level trauma center using the ICD-9 code 431 for sICH. We included adult patients (>18 years of age) with sICH who had a computed tomography of the head (CTH) and an abnormal TEG-PM upon admission and prior to blood product correction along with a repeat TEG-PM and CTH. Patients were excluded if their charts were miscoded, had a mislabeled identification number, or had a lack of repeat TEG-PM. Figure 1 shows the breakdown of eligible subjects. 


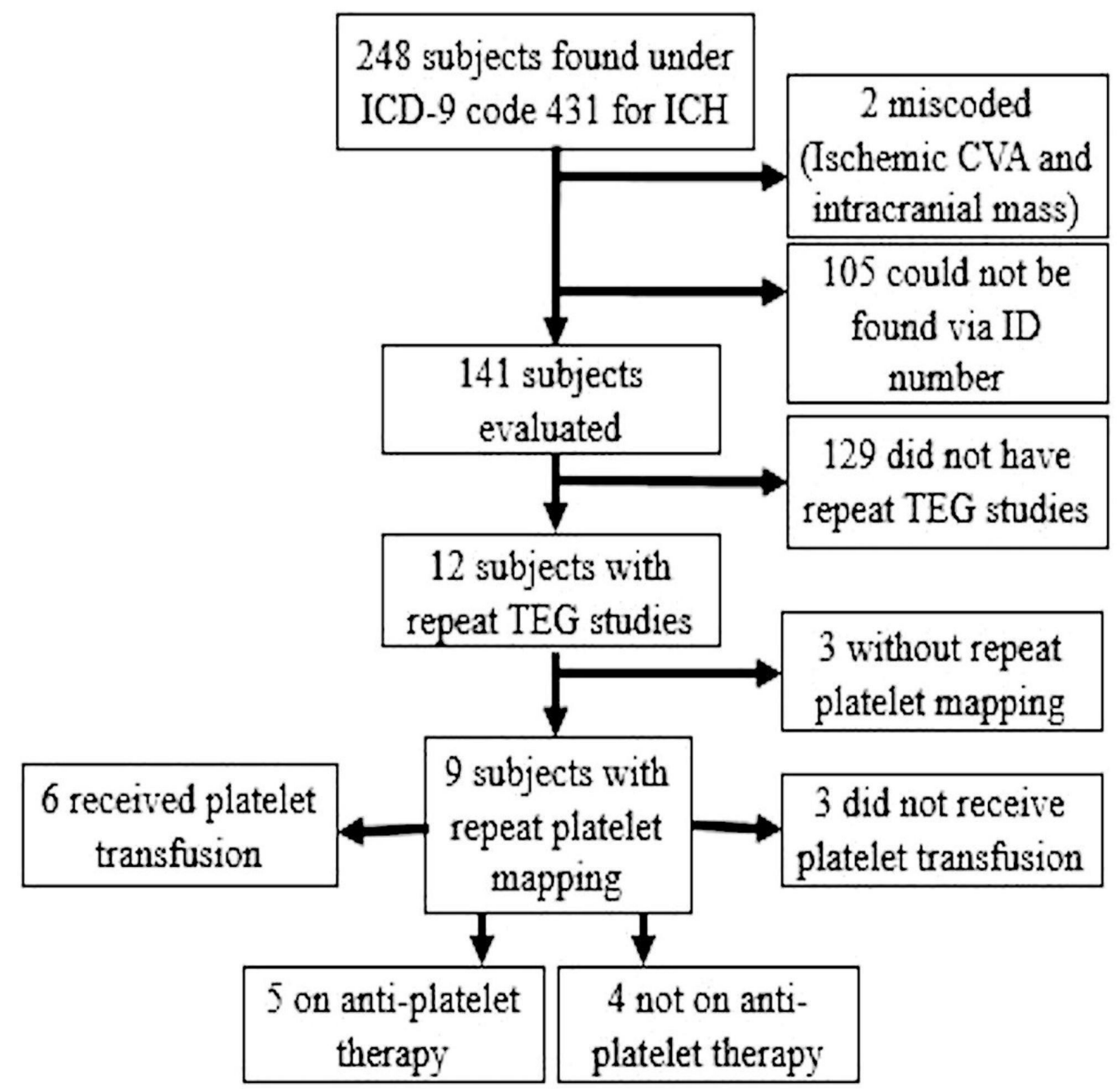

FIGURE 1: Study Design

Baseline characteristics of the sample population included age, sex, weight (kg), comorbidities (hypertension (HTN), hyperlipidemia (HLD), diabetes mellitus (DM), congestive heart failure (CHF), and chronic kidney disease (CKD)), and antiplatelet therapy (none, aspirin, clopidogrel, or dual therapy). sICH related characteristics gathered include Glasgow Coma Score (GCS), National Institute of Health Stroke Score (NIHSS), ICH score, ICH volume from CTHs and subsequent CTHs, units of platelet transfusion, and other blood products received. Deep venous thrombosis (DVT) during hospital stay and the number of inpatient deaths were also collected.

\section{Antithrombotic effect on TEG-PM}

We analyzed ASA, clopidogrel (CLO), and dual antiplatelet therapy (DAPT) usage based on documented patient drug records with abnormal TEG-PM. Considering the local neurosurgical practice and findings of two different studies, a threshold of 50\% inhibition was used as the benchmark for both ADP and AA in this portion of the study in order to determine the sensitivity and specificity of TEG-PM [12-13].

\section{Platelet transfusion effect on TEG-PM}

Decreases in ADP and AA inhibition from the initial lab and the repeat study were analyzed 


\section{Cureus}

between the groups which had or had not received the platelet transfusion. These were then compared to determine the efficacy of TEG-PM for analyzing changes in platelet activity after reversal.

\section{Outcome measurements}

The frequency of hemorrhagic expansion obtained from reviewing the initial and repeat head CT in patients with abnormal TEG-PM results were analyzed. The ICH volume was calculated using the $\mathrm{ABC} / 2$ method, which is a quick method to obtain an accurate estimate of the volume [14]. The effect of platelet transfusion on ICH volume expansion was then determined.

\section{Statistical analysis}

Platelet inhibition is the extent of non-response of the ADP or thromboxane A2 receptor to $\mathrm{ADP}$ and AA, respectively. TEG measures the binding activity of activated platelets to a fibrin mesh and represents the maximal clot strength via measurements of maximal amplitude (MA). Platelet aggregation to agonist can, therefore, be calculated using the following formula: ((MAADP/AA - MAFibrin) / (MAThrombin - MAFibrin) x 100). Percentage platelet inhibition is therefore: $100 \%$ - \% platelet aggregation. Using these formulas, the TEG-PM software calculates ADP \% inhibition and AA \% inhibition. AA is the agonist in the case of ASA therapy, whereas ADP is the agonist in CLO therapy [12]. Categorical data included ASA usage, CLO usage, DAPT, no APT, ADP inhibition of $>50 \%$, AA inhibition of $>50 \%$, initial and repeat ADP inhibition \%, initial and repeat AA inhibition \%, platelet transfusion, no platelet transfusion, and ICH expansion. Response to platelet transfusion was noted if there was at least a $20 \%$ difference from initial and follow-up AA and ADP values. The Fisher's exact test was used to analyze our categorical data using a 2x2 contingency table. Odds ratios and confidence interval analyses were performed using a confidence interval (CI) of 95\%. Continuous data were compared using t-test between the groups who had received and had not received platelet therapy.

All statistical analyses were performed using GraphPad Prism 7 (LaJolla, CA, USA). A p value of $<0.05$ was considered significant.

\section{Results}

Two hundred and forty-eight patients were found to have sICH, but 107 were excluded due to incomplete documentation. Out of the 141 patient charts reviewed, nine of those patients met our inclusion criteria. Continuous variables between the group which had received platelet transfusion and the group which had not received platelet transfusion showed no statistically significant differences $(\mathrm{p}=0.77$; Table 1$)$.

\begin{tabular}{|c|c|c|c|}
\hline & Received platelet therapy & Did not receive platelet therapy & $P$ value \\
\hline Total $(\mathrm{N}=9)$ & 6 & 3 & 1 \\
\hline Mean Age (years) & 68.17(18.08) & 57.33(17.62) & 1 \\
\hline Male & $5(55.6)$ & $2(22.2)$ & 1 \\
\hline Female & 1(11.1) & 1(11.1) & 1 \\
\hline Mean Weight & 78.27(9.28) & 84.47(13.36) & 1 \\
\hline
\end{tabular}




\section{Cureus}

HTN

HLD

DM

Prior CVA

CAD

CKD

Antiplatelet therapy PRE-ICH

ASA only

Clopidogrel only

ASA and clopidogrel

None

$\mathrm{ICH}-$ related characteristics

Median GCS score

Median NIHSS score

Median ICH score

Mean ICH initial volume $(\mathrm{mL})$

Mean initial AA inhibition (\%)

Mean initial ADP inhibition(\%)

Secondary Outcomes

DVT complication during hospital stay

Inpatient deaths
$5(83.3)$

2(66.7)

1

2(33.3)

2(66.7)

1

$1(16.7)$

1(33.3)

1

$1(16.7)$

1(33.3)

1

2(33.3)

0

1

0

0

1

3(50)

1(33.3)

1

0

0

1

$1(16.7)$

0

2(66.7)

9.67(3-15)

$9.67(10-15)$

1

7.25(1-18)

$12.67(2-20)$

1

2.17(1-4)

1(0-3)

1

52.42(45.65)

8.94(6.63)

1

78.5(14.9)

$50.57(45.34)$

1

$50.22(36.2)$

80.77(18.13)

1

1(16.7)

0

1

1(16.7)
0

\section{TABLE 1: Subject Characteristics}

Data are given as $\mathrm{n}(\%$ total), median (range), or mean (+ SD).

Yrs: years; Kg: kilogram; HTN: hypertension; HLD: hyperlipidemia; DM: diabetes mellitus; CVA: cerebrovascular accident; CAD: coronary artery disease; CKD: chronic kidney disease; ASA: aspirin; ICH: intracerebral hemorrhage; GCS: Glasgow coma score; NIHSS: National Institute of Health Stroke Scale; mL: milliliters; AA: arachidonic acid; ADP: adenosine diphosphate; DVT: deep venous thrombosis; NS: nonsignificant.

Antithrombotic effect on TEG-PM: No statistical significance was found on the antithrombotic effects of ASA and CLO on platelet mapping ( $\mathrm{p}=1.00$ for both) (Table 2). 


\section{Cureus}

\begin{tabular}{lllllllll}
\hline & \multicolumn{3}{c}{ Clopidogril Use (N=8) } & \multicolumn{2}{l}{ Aspirin Use (N=8) } \\
\hline & & Yes & No & P value & Yes & No & P value \\
ADP inhibition $>50 \%$ & Yes & 1 & 4 & 1 & NA & NA & NA \\
& No & 0 & 3 & 1 & NA & NA & NA \\
AA inhibition $>50 \%$ & Yes & NA & NA & NA & 4 & 3 & 1 \\
& No & NA & NA & NA & 1 & 0 & 1
\end{tabular}

\section{TABLE 2: Effect of Clopidogrel and Aspirin on ADP and AA Inhibition}

ADP: adenosine diphosphate; AA: arachidonic acid.

Sensitivity and specificity of platelet mapping for CLO was $100 \%$ and $42.9 \%$, respectively, and $80 \%$ and $0 \%$, respectively, for ASA, using the benchmark of 50\% AA and ADP inhibition.

Platelet transfusion effect on TEG-PM: Platelet transfusion did not significantly decrease AA $(\mathrm{p}=1.00, \mathrm{OR}=0.75$, CI 0.04-14.97) or ADP inhibition $(\mathrm{p}=1.00, \mathrm{OR}=0.13, \mathrm{CI} 0.01-3.23)$, per TEGPM (Table 3).

\begin{tabular}{|c|c|c|c|c|c|c|c|}
\hline & & \multicolumn{3}{|c|}{ Platelet Transfusion $(\mathrm{N}=8)$} & \multicolumn{3}{|c|}{ Hemorrhagic Expansion ( $\mathrm{N}=8)$} \\
\hline & & Yes & No & $P$ value & Yes & No & $P$ value \\
\hline \multirow{2}{*}{ Decrease in ADP } & Yes & 1 & 2 & \multirow{2}{*}{1} & 0 & 3 & \multirow{2}{*}{0.5} \\
\hline & No & 4 & 1 & & 2 & 4 & \\
\hline \multirow{2}{*}{ Decrease in $A A$} & Yes & 3 & 2 & \multirow{2}{*}{1} & 1 & 5 & \multirow{2}{*}{1} \\
\hline & NO & 2 & 1 & & 1 & 2 & \\
\hline
\end{tabular}

\section{TABLE 3: Platelet Transfusion or Hemorrhagic Expansion Associated with Reduction with ADP or AA}

ADP: adenosine diphosphate; AA: arachidonic acid.

Outcome measurements: Hemorrhagic expansion on CTH was not associated with a decrease $\mathrm{AA}(\mathrm{p}=1.00, \mathrm{OR}=0.40,95 \% \mathrm{CI} 0.2-10.02)$ or $\mathrm{ADP}(\mathrm{p}=0.50, \mathrm{OR}=0.26,95 \%$ CI $0.01-7.27)$ inhibition per TEGPM, respectively or with platelet transfusion ( $\mathrm{p}=1.00, \mathrm{OR}=0.40,95 \%$ CI $0.2-10.02)$ as seen in Tables 3-4. 


\section{Cureus}

\begin{tabular}{|c|c|c|c|c|c|c|c|}
\hline & & \multicolumn{3}{|c|}{ Hemorrhagic Expansion ( $\mathrm{N}=9$ ) } & \multicolumn{3}{|c|}{ Death/Complications $(\mathrm{N}=9)$} \\
\hline & & Yes & No & $P$ value & Yes & No & $P$ value \\
\hline \multirow{2}{*}{ Platelet Transfusion } & Yes & 1 & 5 & \multirow{2}{*}{1} & 1 & 5 & \multirow{2}{*}{1} \\
\hline & No & 1 & 2 & & 0 & 3 & \\
\hline
\end{tabular}

TABLE 4: Hemorrhagic Expansion or Thrombotic Complications Associated with Platelet Transfusion

Secondary outcomes of DVT complication or inpatient deaths were not associated with platelet transfusion ( $\mathrm{p}=1.00$; Table 4$)$

\section{Discussion}

These results suggest that platelet mapping with thromboelastography is not a clinically useful modality to measure platelet inhibition in sICH patients who were on antithrombotics or to monitor changes in platelet function following platelet transfusion. A large contributing factor to its lack of efficacy appears to be due to its particularly poor specificity. These findings are similar to a recent TEG-PM study in acute trauma patients on APT in which a stricter benchmark of AA and ADP inhibition at 70\% was used, and in a recent study following minor injury [15-16]. No precise level of platelet receptor inhibition has been established to determine clinical significance for platelet dysfunction or normal function due to wide ranges of inhibition seen in non-APT control groups. An AA and ADP inhibition range of $0 \%$ to $10 \%$ and $0 \%$ to $58 \%$, respectively, have been found in healthy blood donors $(n=43)$ [13]. Also, a mean AA and ADP inhibition of $17.5 \%$ and $47.8 \%$ respectively have been found in non-APT pre-operative patients $(n=59)$ [12]. These former findings agree with the lack of specificity and limitation of the TEGPM system to be used independently as a platelet function test in these scenarios. The level of inhibition seen in subjects not on APT suggests that there may be other factors altering platelet function or interacting with platelet receptors. Some factors include altered physiology in trauma, alcohol consumption, certain foods, such as garlic, and temperature changes [11$12,17]$. Increased ADP inhibition (64.5\% vs 15.5\%; $\mathrm{p}<0.01$ ) and AA inhibition (25.6\% vs 2.2\%; $\mathrm{p}<0.01)$ in isolated head injuries unrelated to APT were found compared to an uninjured group [11]. The average ADP inhibition seen with traumatic brain injury (TBI) has been found to be $86.5 \%$ to $93.1 \%$ with a GCS $<8$ and $40.4 \%$ to $49.8 \%$ with a GCS $>8$ compared to $15.5 \%$ of the controls $[11,18]$. This increased level of ADP inhibition with TBI appears to correlate with greater quantities of dense granules seen via flow cytometric assays [19]. In a study of 459 patients with minor injury, there was no correlation of TEG-PM with injury severity score (ISS), length of stay (LOS) or mortality despite the finding of normal clot strength with abnormal AA and ADP inhibition of $30 \%$ and 58\%, respectively [16]. Despite these recent findings on the effects of trauma on platelet receptor inhibition, it is still uncertain as to whether these findings apply to the non-traumatic

spontaneous cerebral injuries. TEG-PM was not compared to other current platelet function tests in this study. VerifyNow-ASA and PFA-100 have been previously evaluated. Forty-two percent of patients assessed with the VerifyNow assay and 52\% assessed with the PFA-100 assay were found to have abnormal platelet function without any history of antiplatelet use. Overall, the agreement between the two assays is poor $(\mathrm{k}=0.26, \mathrm{p}=0.07)$ [9]. Past studies have shown TEG-PM to be comparable to optical platelet aggregometry and superior to PFA-100 in the assessment of ADP receptor function [12]. Further comparison studies would benefit the evaluation of TEG-PM reliability in such context. 
Platelet transfusion, such as what we showed in our study, was not found to be associated with significant changes in sICH volume expansion, suggesting a lack of outcome benefit from receiving a platelet transfusion. This finding contrasts with that by Naidech et al. who found that platelet transfusion improved platelet activity (as measured by the VerifyNow-ASA assay) resulting in a smaller final hemorrhage size [20]. Recently, the PATCH trial, which was a multicenter trial, evaluated platelet transfusion to standard care in patients with ICH who were on APT. One hundred ninety patients were enrolled over 80 months. The primary findings of odds of death or dependence at three months were higher in the platelet-transfused group $(\mathrm{p}=0.01$, OR $2.05,95 \% \mathrm{CI} 1.18-3.56)$ [8]. In a separate study in elderly patients, the transfusion of platelets did not reduce mortality [7]. Given these findings, platelet transfusion cannot be recommended for patients with ICH who were on APT.

This study has expected limitations associated with a retrospective chart review. The determination of APT use was dependent on documented medication records received by the hospital. Many charts were excluded due to errors in coding and lack of repeat TEG-PM studies. Other possible confounders included transfer admissions and direct admissions to the center's emergency department. Approximately one-third of the included subjects were transferred from other hospitals. Additionally, our sample size was small, and future studies with larger populations could help to determine dose-dependent changes of AA and ADP inhibition and other influential factors to their levels on TEG-PM. The lack of significance with the use of TEG-PM following platelet transfusion is even more relevant given that patients who received platelet transfusion had lower NIHSS.

\section{Conclusions}

The use of platelet mapping with thromboelastography appears to be limited by its nonspecific findings of platelet receptor inhibition in determining anti-platelet therapy, determining postplatelet transfusion changes, and predicting hemorrhagic expansion outcomes in patients with sICH. Platelet transfusion cannot be recommended for the sole purpose of APT reversal in ICH patients based on TEG-PM.

\section{Additional Information \\ Disclosures}

Human subjects: Consent was obtained by all participants in this study. MU Institutional review Board issued approval 2004713. Animal subjects: All authors have confirmed that this study did not involve animal subjects or tissue. Conflicts of interest: In compliance with the ICMJE uniform disclosure form, all authors declare the following: Payment/services info: All authors have declared that no financial support was received from any organization for the submitted work. Financial relationships: All authors have declared that they have no financial relationships at present or within the previous three years with any organizations that might have an interest in the submitted work. Other relationships: All authors have declared that there are no other relationships or activities that could appear to have influenced the submitted work.

\section{References}

1. Sacco S, Marini C, Toni D, Olivieri L, Carolei A: Incidence and 10-year survival of intracerebral hemorrhage in a population-based registry. Stroke. 2009, 394-399. 10.1161/STROKEAHA.108.523209

2. Broderick JP, Brott TG, Duldner JE, Tomsick T, Huster G: Volume of intracerebral hemorrhage: a powerful and easy-to-use predictor of 30-day mortality. Stroke. 1993, 24:987993. 10.1161/01.STR.24.7.987 
3. Hanley JP: Warfarin reversal. J Clin Pathol. 2004, 57:1132-1139. 10.1136/jcp.2003.008904

4. Thompson BB, Béjot Y, Caso V, et al.: Prior antiplatelet therapy and outcome following intracerebral hemorrhage: a systematic review. Neurology. 2010, 75:1333-1342. 10.1212/WNL.0b013e3181f735e5

5. Saloheimo P, Ahonen M, Juvela S, Pyhtinen J, Savolainen ER, Hillbom M: Regular aspirin-use preceding the onset of primary intracerebral hemorrhage is an independent predictor for death. Stroke. 2006, 37:129-133. 10.1161/01.STR.0000196991.03618.31

6. Hemphill JC, Greenberg SM, Anderson CS, et al.: Guidelines for the management of spontaneous intracerebral hemorrhage. Stroke. 2015, 46:2032-2060.

10.1161/STR.0000000000000069

7. Downey DM, Monson B, Butler KL, et al.: Does platelet administration affect mortality in elderly head-injured patients taking antiplatelet medications?. Am Surg. 2009, 75:1100-1103.

8. Baharoglu MI, Cordonnier C, Salman RAS, et al.: Platelet transfusion versus standard care after acute stroke due to spontaneous cerebral haemorrhage associated with antiplatelet therapy (PATCH): a randomised, open-label, phase 3 trial. The Lancet. 2016, 387:2605-2613. 10.1016/S0140-6736(16)30392-0

9. Naidech AN, Jovanovic B, Liebling S, et al.: Reduced platelet activity is associated with early clot growth and worse 3-month outcome after intracerebral hemorrhage. Stroke. 2009, 40:2398-2401. 10.1161/STROKEAHA.109.550939

10. Paniccia R, Priora R, Alessandrello Liotta A, Abbate R: Platelet function tests: a comparative review. Vasc Health Risk Manag. 2015, 11:133-148. 10.2147/VHRM.S44469

11. Castellino FJ, Chapman MP, Donahue DL, et al.: Traumatic brain injury causes platelet adenosine diphosphate and arachidonic acid receptor inhibition independent of hemorrhagic shock in humans and rats. J Trauma Acute Care Surg. 2014, 76:1169-1176. 10.1097/TA.0000000000000216

12. Collyer TC, Gray DI, Sandhu R, Berridge J, Lyons G: Assessment of platelet inhibition secondary to clopidogrel and aspirin therapy in preoperative acute surgical patients measured by thrombelastography platelet mapping. Br J Anaesth. 2009, 102:492-498. 10.1093/bja/aep039

13. Bochsen L, Wiinberg B, Kjelgaard-Hansen M, Steinbrüchel DA, Johansson PI: Evaluation of the TEG ${ }^{\circledR}$ platelet mappingTM assay in blood donors. Thrombosis Journal. 2007, 5:3-5. 10.1186/1477-9560-5-3

14. Kothari RU, Brott T, Broderick JP, et al.: The ABCs of measuring intracerebral hemorrhage volumes. Stroke. 1996, 27:1304-1305. 10.1161/01.STR.27.8.1304

15. Daley MJ, Trust MD, Peterson EJ, et al.: Thromboelastography does not detect preinjury antiplatelet therapy in acute trauma patients. Am Surg. 2016, 2:175-180.

16. Sirajuddin S, Valdez C, DePalma L, et al.: Inhibition of platelet function is common following even minor injury. J Trauma Acute Care Surg. 2016, 81:328-332. 10.1097/TA.0000000000001057

17. Van Poucke S, Stevens K, Marcus AE, Lancé M: Hypothermia: effects on platelet function and hemostasis. Thrombosis Journal. 2014, 12:31. 10.1186/s12959-014-0031-z

18. Davis PK, Musunuru H, Walsh M, et al.: Platelet dysfunction is an early marker for traumatic brain injury-induced coagulopathy. Neurocrit Care. 2013, 18:201-208. 10.1007/s12028-0129745-6

19. Bartels AN, Johnson C, Lewis J, et al.: Platelet adenosine diphosphate inhibition in trauma patients by thromboelastography correlates with paradoxical increase in platelet dense granule content by flow cytometry. Surgery. 2016, 160:954-959. 10.1016/j.surg.2016.07.012

20. Naidech AM, Liebling SM, Rosenberg NF, et al.: Early platelet transfusion improves platelet activity and may improve outcomes after intracerebral hemorrhage. Neurocrit Care. 2012, 16:82-87. 10.1007/s12028-011-9619-3 